\title{
Implantes cono Morse con sistema de indexación interno. Descripción del caso clínico
}

\author{
Morse taper interface with prosthetic index. Clinical case presentation
}

\author{
Thomé $\mathrm{G}^{1}$, Bernardes $\mathrm{SR}^{2}$, Castro $\mathrm{CG}^{3}$, Golin $\mathrm{AL}^{4}$, Sartori IAM ${ }^{5}$
}

\begin{abstract}
RESUMEN
El empleo de implantes cono Morse con indexadores para instalación con torque interno sería una opción interesante para el diseño de un implante dentario, que incluso podría traer más opciones protéticas a ese sistema ya que el índex interno también puede utilizarse como una referencia para las restauraciones. El objetivo de este artículo es describir un caso clínico con empleo de implantes cono Morse y un sistema de indexación interno, que evitaría el uso de un montador externo que ya venga adaptado de fábrica. La técnica de cirugía sin colgajo asociada al implante cono Morse con indexador hexagonal interno demostró ser un procedimiento seguro para rehabilitación del área edéntula posterior del maxilar.
\end{abstract}

Rev. Clin. Periodoncia Implantol. Rehabil. Oral Vol. 4(2); 69-73, 2011.

Palabras clave: Implantes dentarios, conexiones tipo Morse, torque interno.

\begin{abstract}
The use of Morse Taper implants with index to the installation with internal torque should be an interesting option to the design of a dental implant, It could even bring more prosthetic solutions to this system by the fact that the internal index could also be used as a reference for restorations. This article aims to describe a clinical case with the use of Morse Taper implants and a system of internal index, avoiding the use of an external mount, which already comes adapted from factory. The technique of flapless surgery associated to the Morse Taper implant with internal hex index demonstrated to be a safe procedure to the rehabilitation of the posterior edentulous region in the jaw.
\end{abstract}

Rev. Clin. Periodoncia Implantol. Rehabil. Oral Vol. 4(2); 69-73, 2011.

Key words: Verrucous carcinoma, case report.

\section{INTRODUCCIÓN}

Durante los últimos años, los implantes dentarios han sido sometidos a torques internos en el momento de su instalación quirúrgica. Esa característica fue una decisión tomada por los fabricantes de implantes dentarios porque los implantes con torques internos permiten una instalación quirúrgica precisa, seguridad ante deformaciones plásticas en la aplicación final del torque, facilidad para la colocación del implante e integridad de la interfaz pilar/implante, sin consecuencias futuras en relación a la prótesis ${ }^{(1-4)}$. Así, muchos cirujanos dentistas han evitado utilizar los montadores prefabricados que acompañan al implante de fábrica porque ese tipo de pieza podría dificultar el proceso de instalación del implante.

Consecuentemente, diferentes interfaces pilar/implante fueron desarrolladas en función de éste y otros motivos, tales como mayor seguridad y facilidad en los procedimientos durante la etapa protética y mayor estabilidad del sistema de retención pilar/implante ${ }^{(1,5-18)}$. Además, desde el punto de vista mecánico, la unión entre el implante y su componente protético pasó a desempeñar un rol importante para los resultados clínicos y biológicos. Movimientos entre el implante y los pilares en dos piezas podrían generar la formación de una microhendidura entre las partes ${ }^{(19)}$, lo que podría resultar en la contaminación interna del implante ${ }^{(20,21)}$. Conceptos como "platform switch" pasaron a ser descritos por la literatura moderna como factores importantes para la consecución de la estética y el mantenimiento del tejido periimplantario a largo plazo ${ }^{(22-29)}$. De acuerdo con los conceptos presentados, se cree que los implantes con interfaz cono Morse presentarían un menor potencial de pérdida ósea periimplantaria cuando se utilizan de manera correcta ${ }^{(24,30-33)}$. Por lo tanto, el empleo de implantes cono Morse con indexadores para instalación con torque interno sería entonces una opción interesante para el diseño de un implante dentario.

El objetivo de este artículo es describir un caso clínico con empleo de implantes cono Morse y un sistema de indexación interno, lanzado recientemente al mercado de implantes dentales. Fue realizada una revisión crítica de la literatura centrada en los aspectos relacionados a la planificación y ejecución de los casos presentados, con base en el paso a paso de la rehabilitación de este caso.

\section{DESCRIPCIÓN DEL CASO CLÍNICO}

Paciente compareció a la selección del Instituto Latinoamericano de Investigación y Enseñanza Odontológica (ILAPEO, Curitiba, Brasil) presentando ausencias dentarias posteriores (Figuras 1 y 2). La queja principal del paciente se refería a la ausencia del elemento 16. Tras el examen clínico (Figura 1) y tomográfico (Figura 2), se observó un gran volumen óseo en el sentido vestíbulolingual en esta región. La planificación de elección fue la rehabilitación con prótesis fija sobre implante, siendo la instalación del implante realizada en una cirugía sin colgajo. La técnica de cirugía flapless ha sido indicada como un procedimiento seguro y previsible en los casos en que la selección del paciente y la ejecución de la técnica sean apropiadas ${ }^{(34)}$.

\footnotetext{
1. Ph.D en Implantología Dental. São Leopoldo Mandic en Campinas/SP. Presidente del Grupo Neodent. Curitiba/PR, Brasil.

2. Ph.D en Rehabilitación Oral. USP, Ribeirão Preto/SP. Profesor del Curso de Especialización en Prótesis Dental del ILAPEO. Curitiba/PR, Brasil.

3. Máster en Odontología. UFU, Uberlândia/MG. Profesora Auxiliar del ILAPEO. Curitiba/PR, Brasil.

4. Máster en Ingeniería Mecánica. PUC, Curitiba/PR. Coordinador de Ingeniería de Neodent. Curitiba/PR, Brasil.

5. Ph.D en Rehabilitación Oral. USP, Ribeirão Preto/SP. Coordinadora del Curso de Especialización en Prótesis Dental y de la Maestría en Odontología en el Área de Implantología Dental del ILAPEO. Curitiba/PR, Brasil.
} 


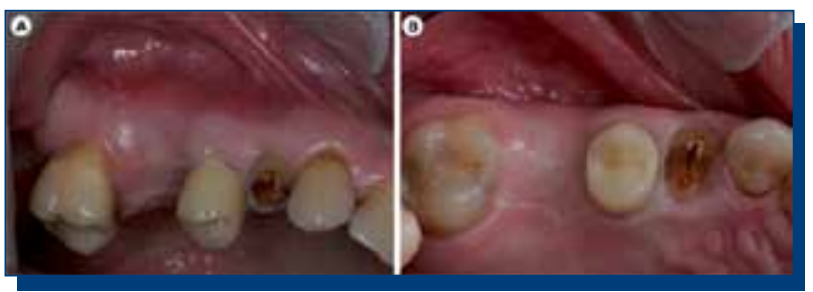

Figura 1. Caso clínico inicial. Vista vestibular (A) y oclusal (B) de la región del diente 16.

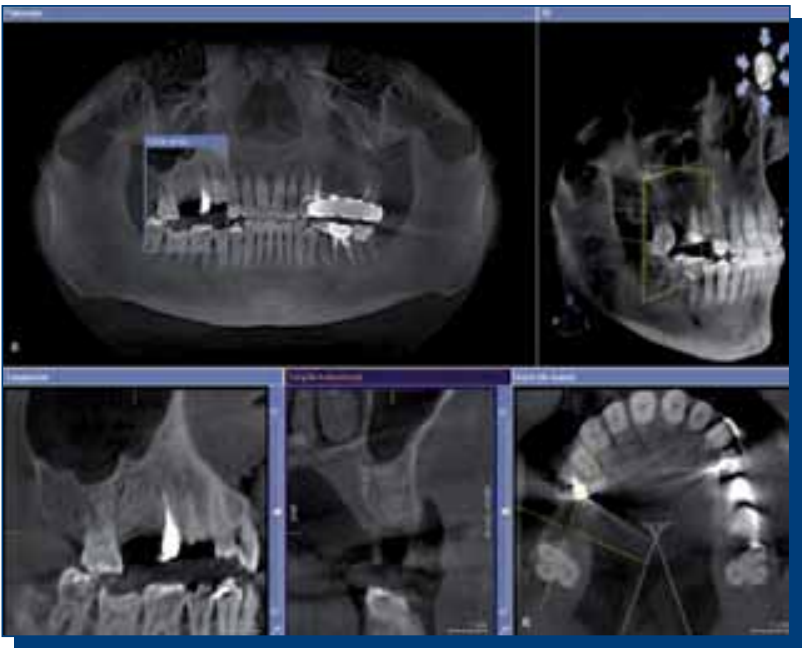

Figura 2. Tomografía computadorizada de la región del diente 16 (Corte coronal panorámico, parasagital, axial y $3 \mathrm{D}$ )

La instrumentación quirúrgica fue iniciada con la Broca Lanza (Neodent ${ }^{\mathrm{TM}}$, Curitiba, Brasil) (Figura $3 \mathrm{~A}$ ), seguida de la Broca 2.0 (Neodent ${ }^{\mathrm{TM}}$ ). Tras su inserción se hizo una radiografía periapical (Figura 3B) confirmando la posición y el eje de inserción que se seguirá. Con el Bisturí Circular (Neodent ${ }^{\mathrm{TM}}$ ) se removió una porción de la mucosa oclusal (Figuras 3C, 3D y 3E). Esta es la única incisión realizada durante toda la cirugía. La perforación fue continuada respetando la secuencia de brocas -2.0 y 3.5 (Figuras $4 \mathrm{~A}$ y $4 \mathrm{~B}$ ) para el implante previamente seleccionado (Alvim Cono Morse 4.3, Neodent ${ }^{\mathrm{TM}}$ ) (Figura 5). La opción del implante Alvim CM fue seleccionada en función de su característica compactadora indicada para hueso tipo III y IV (informaciones del fabricante). La realización de la osteometría con el posicionador radiográfico (Figura 4C) confirmó la posibilidad de instalación infraósea del implante con extensión de $13 \mathrm{~mm}$ (Figura 5).

Al abrir el implante en el envoltorio (Figura 5), se nota la ausencia de montador, ya que la línea de implantes con indexador hexagonal interno no posee esa pieza. Se utilizó una conexión cono Morse $(\mathrm{CM})$, con indexador para la captura del implante. La empresa ofrece las conexiones para contra ángulo o trinquete. Para la correcta captura del implante, la conexión cono Morse deberá ser presionada contra el implante posicionado dentro del cristal. Luego de verificada la adaptación de la llave, el implante fue conducido al alvéolo quirúrgico.

El implante fue instalado con torque de inserción mayor a 80N. $\mathrm{cm}$. La bioingeniería de este sistema de conexión, el cual prescinde del uso de montador, permite una alta resistencia al torque de inserción. Inmediatamente después se realizó una radiografía periapical (Figuras $6 \mathrm{~A}, 6 \mathrm{~B}$ y $6 \mathrm{C}$ ). Se observa la preservación de los tejidos periimplantarios adyacentes, característica de la técnica flapless (Figura 7A). En este caso, se optó por el empleo de carga inmediata debido al valor de estabilidad primaria alcanzada (Figura 6C). Se utilizó el nuevo Kit de Selección Protética CM (Neodent ${ }^{\mathrm{TM}}$ ) al finalizar la instalación de los implantes. El conjunto de réplicas de componentes ayuda al profesional en la selección de la altura de la cinta de los componentes (Figura 7B). El extremo de la prótesis debe estar apartado al menos $1.5 \mathrm{~mm}$ del tejido óseo para evitar una posible pérdida (Figuras 7C y 7D), debido al restablecimiento de un espacio biológico en el surco gingival periimplantario. La corona provisoria fue cementada (Figura 8) y tras 1 mes (Figura 9), se realizó el acompañamiento del caso. La restauración final se instalará después de una evaluación de los aspectos del caso clínico, relacionados tanto con los pacientes como con los signos y síntomas detectados en la región.

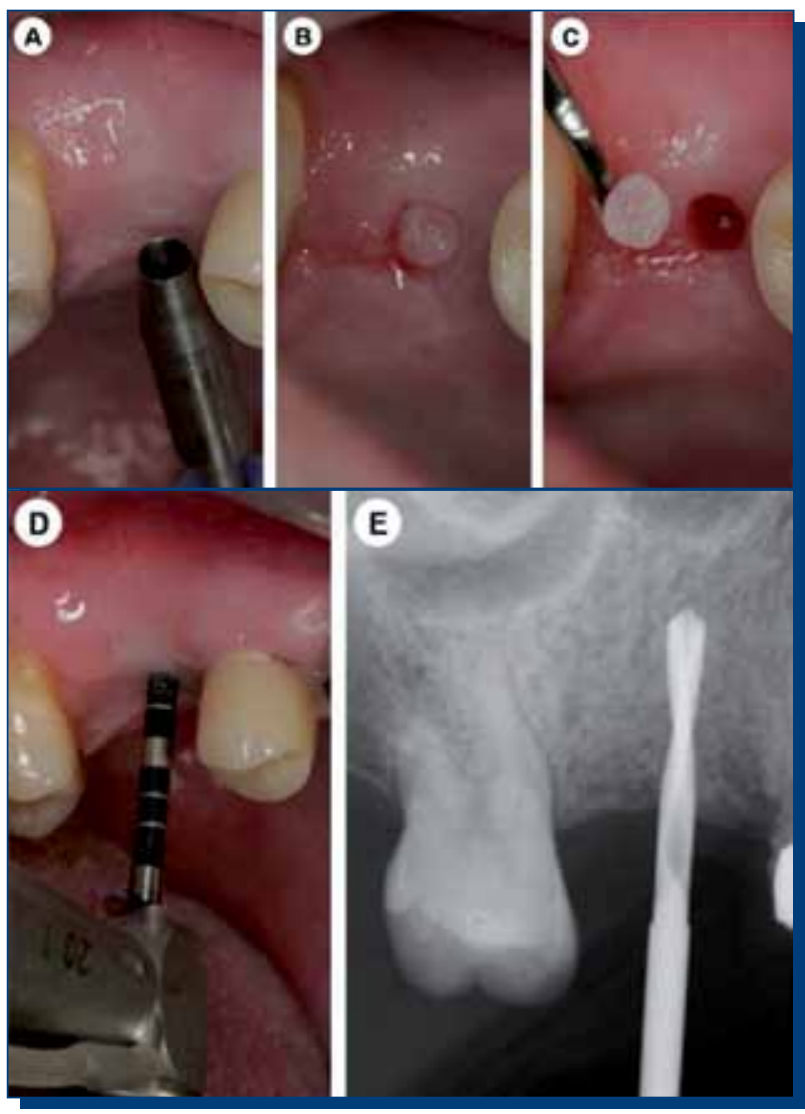

Figura 3. A. Broca lanza; B. Vista radiográfica de la broca lanza en el reborde óseo; C. Bisturí circular; D y E. Remoción de mucosa por medio del bisturí circular.
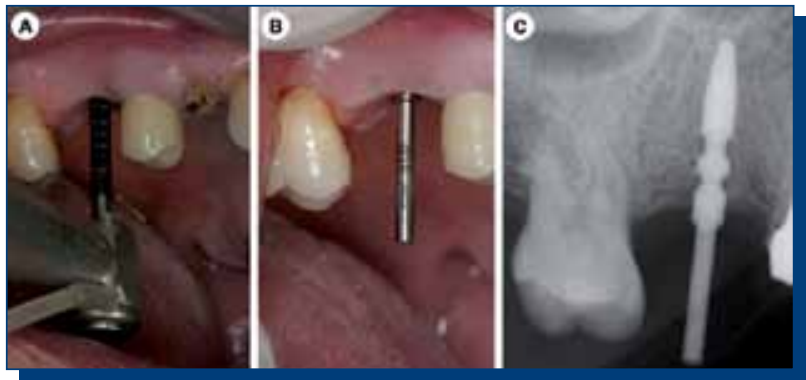

Figura 4. A y B. Perforación con broca 3.5; B y C. Osteometría con posicionador radiográfico.

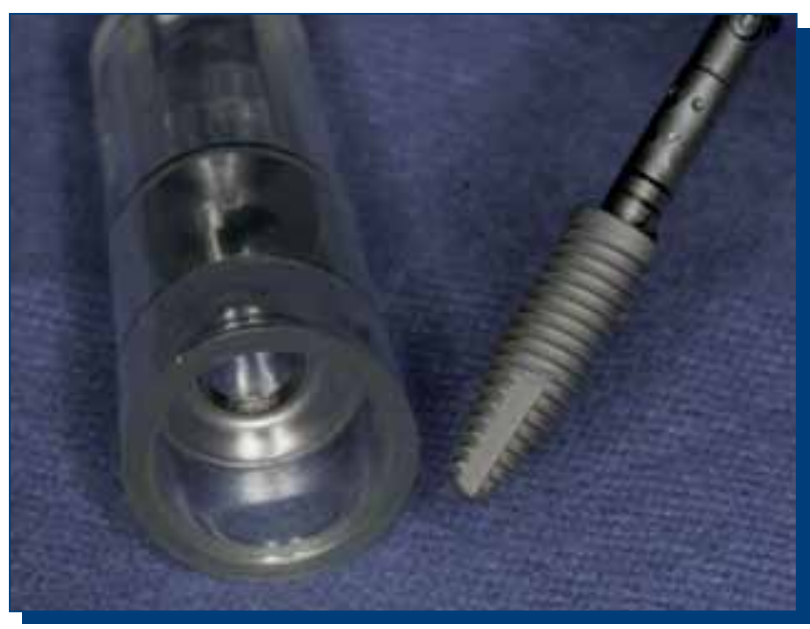

Figura 5. Implante Alvim CM con indexador hexagonal interno (Alvim CM 4.3×13, Neodent ${ }^{\mathrm{TM}}$ ). 


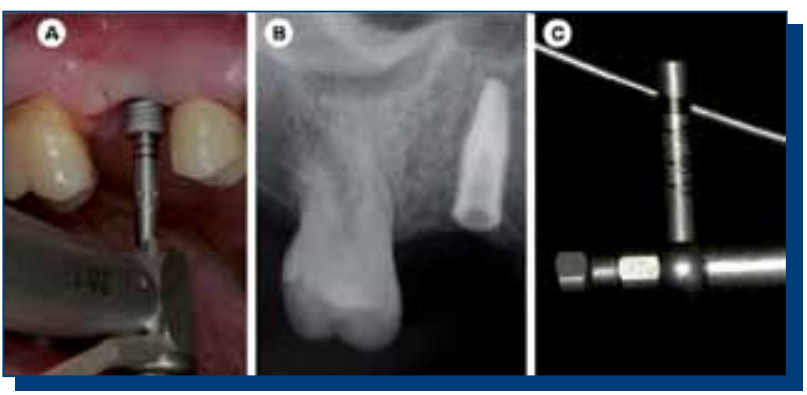

Figura 6. A. Instalación del implante con conexión CM para contra ángulo; B. Aspecto radiográfico del implante instalado; $\mathbf{C}$. Indicación de la estabilidad primaria alcanzada.

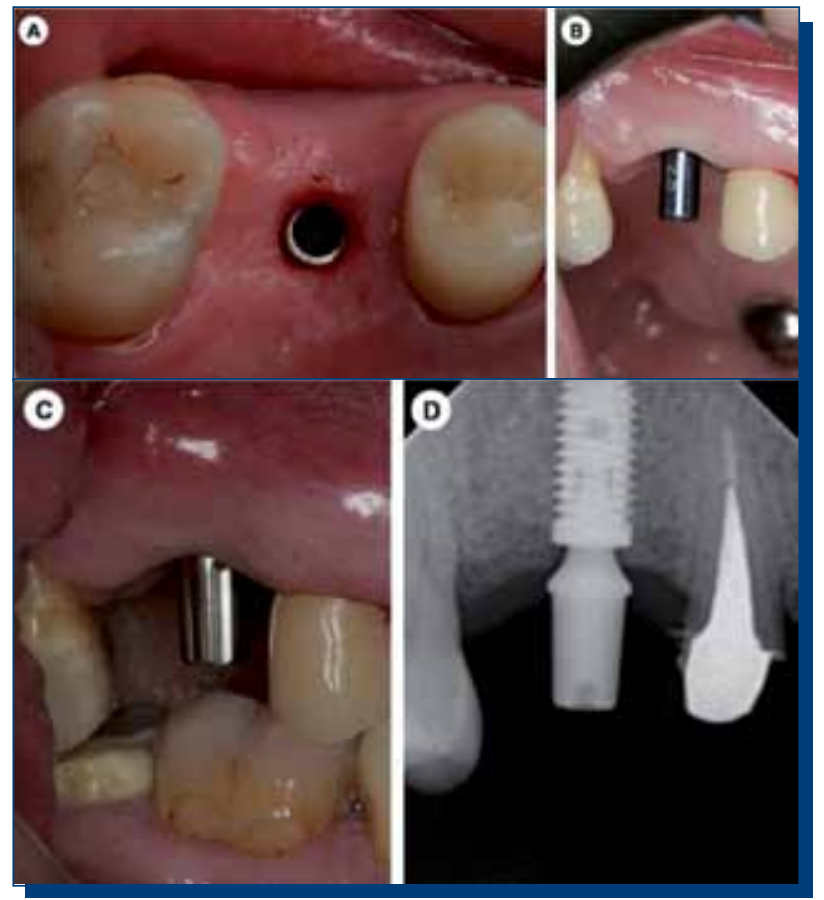

Figura 7. A. Vista oclusal del implante instalado; B. Selección del muñón; C. Muñón instalado; D. Aspecto radiográfico de la porción cervical del implante con el muñón instalado.

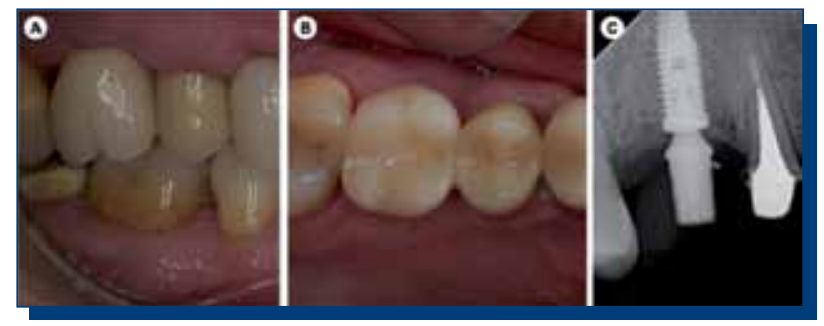

Figura 8. Corona provisoria cementada. A. Aspecto clínico vestibular y B. Oclusal; C. Aspecto radiográfico.

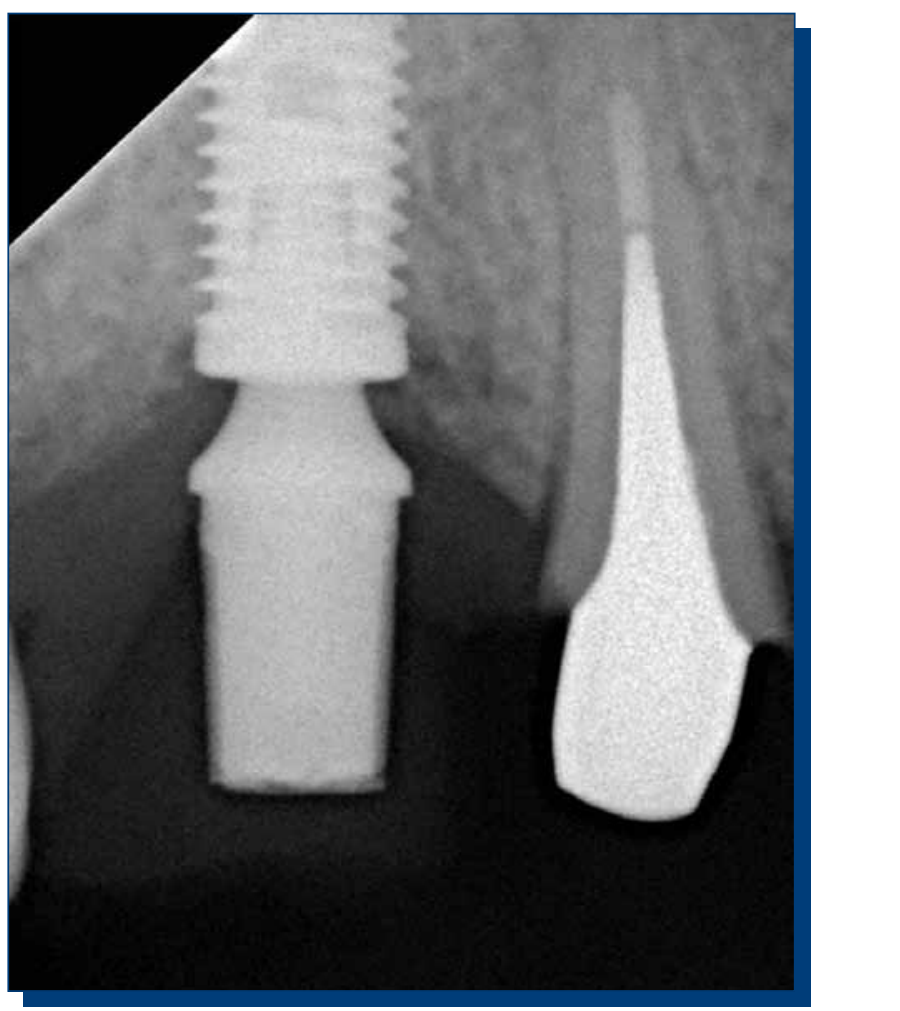

Figura 9. Aspecto radiográfico tras 1 mes de acompañamiento.

\section{DISCUSIÓN}

Para la resolución de casos con carga inmediata del implante, la estabilidad primaria es el principal factor que se considerará. Clínicamente, la estabilidad primaria se verifica por el torque de inserción obtenido al finalizar la instalación. Los valores de torque alcanzados están relacionados con la característica del sitio de instalación, como la baja calidad ósea y también con el diseño del implante. En el caso clínico presentado, se alcanzó un valor mayor a 80N. cm (Figura 6C). La utilización en este estudio del implante Alvim CM con indexador confirmó los datos relatados de la posibilidad de obtener una alta estabilidad primaria en hueso de baja calidad y el diseño interno del indexador y de la llave permitieron la instalación manteniendo la integridad tanto del implante como de la llave. El aumento de la resistencia de los implantes en la utilización de torques internos es un hecho que ya se confirmó en estudios comparativos en implantes de hexágono externo con y $\sin$ montadores ${ }^{(35)}$. En ese mismo estudio, cuando se analizan hexágonos de implantes tras la simulación de torque de instalación, el modelo torque interno presentó una geometría que permite una mejor distribución de las tensiones, disminuyendo así las tensiones máximas, y permaneciendo muy abajo de la tensión límite de escurrimiento del titanio grado IV. Las características observadas durante la instalación del implante se corroboran con datos de otros estudios que describieron la instalación quirúrgica precisa, seguridad ante deformaciones plásticas en la aplicación final del torque, facilidad para la colocación del implante e integridad de la interfaz pilar/implante, sin consecuencias futuras en relación a la prótesis cuando se utilizan diseños de torque interno ${ }^{(1-4)}$.

Además, el advenimiento de los implantes con conexiones del tipo cónicas internas permite la instalación subcrestal de los implantes. En un estudio basado en elementos finitos ${ }^{(36)}$ para evaluar la influencia de la extensión del implante y del anclaje bicortical en la distribución de las cargas, los autores concluyeron que, dado que la porción cervical del implante está comprendida por hueso cortical, esto puede ser un factor favorable para los implantes cortos en hueso más medular, pues la rigidez proporcionada por esa situación provocaría que una instalación exitosa dependiese menos de la extensión del implante. Además de los factores mecánicos involucrados, diversas ventajas biológicas como mayor impermeabilidad a la biopelícula bacteriana han justificado la conclusión de que los implantes que presentan las conexiones tipo Morse deben ser la primera opción para la reposición de los dientes ausentes ${ }^{(37)}$.

Pensando en el aspecto protético de ese nuevo sistema, el 
desarrollo de la llave específica con encaje hexagonal que acompaña al implante desde su captura (Figura 5), permite la visualización de la posición vestibular del implante por medio de sus 6 marcas, orientando la colocación de una de las partes planas del hexágono en la vestibular, lo que garantiza la instalación correcta del futuro intermediario. El indexador presente también se podrá utilizar para orientar la instalación de la prótesis en los casos en que sea necesaria la preparación de los intermediarios en el laboratorio, prescindiendo de la guía de resina acrílica para transferencia del intermediario desde el modelo hacia la boca, necesaria para garantizar la correspondencia. Este sistema podría incluso proporcionar más opciones protéticas ya que el índex interno también se puede utilizar como una referencia para las restauraciones. Ese concepto no se ha utilizado en este caso descrito, ya que el correcto posicionamiento del implante ha permitido la utilización del muñón universal sin indexador.

Para alcanzar una rehabilitación exitosa, se debe tener cuidado principalmente con la estabilidad de los tejidos periimplantarios involucrados. Esta estabilidad está relacionada a diferentes factores, que incluyen desde la selección correcta del paciente hasta la confección e instalación de la prótesis tras la cirugía. En general, el origen de la pérdida ósea periimplantaria sería multifactorial(38). Se deben considerar los factores involucrados tanto en la bioingeniería del implante y de la prótesis, como en las características del sitio receptor. Por ejemplo, algunos autores sugieren cambios en el protocolo referente a la distancia entre los implantes dentarios que podría llegar hasta $2 \mathrm{~mm}$ entre las fijaciones tipo cono Morse ${ }^{(27-29)}$. En lo que se refiere al soporte óseo, se puede sugerir que los implantes cono Morse necesitan de una área de protección y soporte óseo de, al menos, $1 \mathrm{~mm}$ a su alrededor para obtener mejores resultados clínicos. El posicionador radiográfico presentado favorece mucho la colocación del implante en relación al hueso siguiendo esas orientaciones. Como es radiopaco y posee marcas correspondientes a la extensión de los implantes disponibles, permite al cirujano calcular correctamente la extensión que debe escogerse para asegurar el posicionamiento subcrestal, lo que ya fue demostrado como más favorable para la consecución del mantenimiento de los tejidos periimplantarios ${ }^{(28,29)}$

Se puede afirmar que el margen vestibular alrededor del implante depende tanto de la altura de la tabla ósea vestibular ${ }^{(39)}$ como del espesor ${ }^{(40,41)}$ y de la calidad ${ }^{(42)}$ del tejido gingival. En lo que se refiere a la mucosa, la forma y el espesor del tejido alrededor de la corona clínica, definidas como biotipo gingival, han sido evaluadas durante la decisión clínica. El riesgo potencial de recesión del tejido y/o la presencia de sombreado grisáceo en la porción cervical son dos desventajas relacionadas con el biotipo gingival fino(40,43). Como el tejido gingival es el retrato de la condición ósea adyacente, fue adoptada la opción de utilizar la técnica de cirugía flapless y el componente plataforma switch con la intención de la preservación del margen óseo. La instalación inmediata del intermediario protético al final de la cirugía, como sucede en el caso clínico presentado, así como el mantenimiento de este componente a lo largo de los años en posición, la instalación infraósea del implante entre 1 a $2 \mathrm{~mm}$, y la correcta localización del punto de contacto, fueron realizadas con el objetivo de obedecer a los requisitos ya mencionados para obtener la estabilidad biológica de los tejidos periimplantarios ${ }^{(28,29,44,48)}$.

\section{CONCLUSIONES}

El resultado exitoso alcanzado en el caso clínico presentado, considerando las limitaciones descritas, se debe a diversos factores, entre ellos, la selección del paciente con volumen óseo adecuado y la correcta planificación y ejecución de la técnica, de acuerdo con las indicaciones del fabricante.

\section{REFERENCIAS BIBLIOGRÁFICAS}

1. Merz BR, Hunenbart S, Belser UC. Mechanics of the implant-abutment connection: An 8-degree taper compared to a butt joint connection. Int $J$ Oral Maxillofac Implants, 2000; 15(4): 519-526.

2. Widmark G, Friberg B, Johansson B, Sindet-Pedersen S Taylor Å. Mk III: A third generation of the self-tapping Brånemark System ${ }^{\circledR}$ Implant, including the new stargrip internal grip design. A 1-year prospective four-center study. Clinical Implant Dentistry and Related Research, 2003; 5(4): 273-279.

3. Khraisat A, Abu-Hammad O, Al-Kayed AM, Dar-Odeh N. Stability of the implant/abutment joint in a single external-hexagon implant system: Clinical and mechanical review. Clinical Implant Dentistry and Related Research, 2004; 6(4): 222-229.

4. Davi LR, Golin AL, Rocha Bernardes S, Amaral de Araújo C, Domingues Neves F. In vitro integrity of implant external hexagon after application of surgical placement torque simulating implant locking. Braz Oral Res, 2008; 22(2): 125-131.

5. Binon PP, Sutter F, Beaty K, Brunski J, Gulbransen H, Weiner R. The role of screw in implant systems. Int J Oral Maxillofac Implants, 1994; 9(supply 1): 48-63.

6. Palmer RM, Smith BJ, Palmer PJ, Floyd PD. A prospective study of Astra single tooth implants. Clin Oral Impl Res, 1997; 8(3): 173-179.

7. Mc Glumphy EA, Mendel DA, Holloway JA. Implant screw mechanics. Dent Clin North Am, 1998; 42(1): 71-89.

8. Levine RA, Clem DS, Wilson Jr TG, Higginbottom F, Saunders SL. A multicenter retrospective analysis of the ITI implant system used for single-tooth replacements: Results of loading for 2 or more years. Int $J$ Oral Maxillofac Implants, 1999; 14(4): 516-520.

9. Mangano C, Bartolucci EG. Single tooth replacement by Morse taper connection implants: A retrospective study of 80 implants. Int J Oral Maxillofac Implants, 2001; 16(5): 675-680.

10. Norton MR. Biologic and mechanical stability of single-tooth implants: 4- to 7-year follow-up. Clin Implant Dent Relat Res, 2001; 3(4): 214-220.

11. Bozkaya $D$, Müftü $S$. Mechanics of the tapered interface fit in dental implants. J Biomech, 2003; 36(11): 1649-1658.

12. Finger IM, Castellón P, Block M, Elian N. The evolution of external and internal implant/abutment connections. Pract Proced Aesthet Dent, 2003; 15(8): 625-632.

13. Abboud M, Koeck B, Stark H, Wahl G, Paillon R. Immediate loading of single-tooth implants in the posterior region. Int $J$ Oral Maxillofac Implants, 2005; 20(1): 61-68.
14. Akour SN, Fayyad MA, Nayfeh JF. Finite element analyses of two antirotational designs of implant fixtures. Implant Dent, 2005 Mar; 14(1): 77-81

15. Bozkaya $D$, Müfü $S$. Mechanics of the taper integrated screwed-in (TIS) abutments used in dental implants. Journal of Biomechanics, 2005 38(1): 87-89.

16. Wennström JL, Ekestubbe A, Gröndahl K, Karlsson S, Lindhe J. Implant-supported single-tooth restorations: A 5-year prospective study. $J$ Clin Periodontol, 2005 Jun; 32(6): 567-574.

17. Norton MR. Multiple single-tooth implant restorations in the posterior jaws: Maintenance of marginal bone levels with reference to the implantabutment microgap. Int J Oral Maxillofac Implants, 2006; 21(5): 777-784. 18. Nordin T, Jönsson G, Nelvig P, Rasmusson L. The use of a conical fixture design for fixed partial prostheses. A preliminary report. Clin Oral Implants Res, 1998; 9(5): 343-347.

19. Rack A, Rack T, Stiller M, Riesemeier H, Zabler S, Nelson K. In vitro synchrotron-based radiography of micro-gap formation at the implantabutment interface of two-piece dental implants. J Synchrotron Radiat 2010; 17(2): 289-294.

20. Jansen VK, Conrads G, Richter EJ. Microbial leakage and marginal fit of the implant-abutment interface. Int J Oral Maxillofac Implants, 1997; 12(4): 527-540.

21. Steinebrunner L, Wolfart S, Bössmann K, Kern M. In vitro evaluation of bacterial leakage along the implant-abutment interface of different implant systems. Int J Oral Maxillofac Implants, 2005; 20(6): 875-881.

22. Gardner DM. Platform switching as a means to achieving implant esthetics. N Y State Dent J, 2005; 71(3): 34-37.

23. Lazzara RJ, Porter SS. Platform switching: A new concept in implant dentistry for controlling postrestorative crestal bone levels. Int $J$ Periodontics Restorative Dent, 2006; 26(1): 9-17.

24. Weng D, Nagata MJ, Bell M, Bosco AF, de Melo LG, Richter EJ. Influence of microgap location and configuration on the periimplant bone morphology in submerged implants. An experimental study in dogs. Clin Oral Implants Res, 2008; 19(11): 1141-1147.

25. Romanos GE, Nentwig GH. Immediate functional loading in the maxilla using implants with platform switching: Five-year results. Int $J$ Oral Maxillofac Implants, 2009; 24(6): 1106-1112.

26. Degidi M, Piattelli A, Shibli JA, Strocchi R, lezzi G. Bone Formation around a dental implant with a platform switching and another with a 
tissue care connection: A histologic and histomorphometric evaluation in man. TITANIUM, 2009; 1(1): 8-15.

27. de Oliveira RR, Novaes AB Jr, Taba M Jr, Papalexiou V, Muglia $V A$. Bone remodeling adjacent to Morse cone-connection implants with platform switch: A fluorescence study in the dog mandible. Int $J$ Oral Maxillofac Implants, 2009; 24(2): 257-266.

28. Novaes AB Jr, Barros RR, Muglia VA, Borges GJ. Influence of interimplant distances and placement depth on papilla formation and crestal resorption: A clinical and radiographic study in dogs. J Oral Implantol, 2009; 35(1): 18-27.

29. Barros RR, Novaes AB Jr, Muglia VA, lezzi G, Piattelli A. Influence of interimplant distances and placement depth on peri-implant bone remodeling of adjacent and immediately loaded Morse cone connection implants: A histomorphometric study in dogs. Clin Oral Implants Res, 2010; 21(4): 371-378.

30. Morris HF, Winkler S, Ochi S, Kanaan A. A new implant designed to maximize contact with trabecular bone: Survival to 18 months. J Oral Implantol, 2001; 27(4): 164-173.

31. Mangano C, Mangano F, Piatelli A, Lezzi G, Mangano A, La Colla L, Mangano A. Single-tooth Morse taper connection implants after 1 year of functional loading: A multicentre study on 302 patients. Eur $J$ Oral Implantol, 2008; 1(4): 305-315.

32. Mangano C, Mangano F, Piattelli A, lezzi G, Mangano A, La Colla L. Prospective clinical evaluation of 1920 Morse taper connection implants: Results after 4 years of functional loading. Clin Oral Implants Res, 2009; 20(3): 254-261.

33. Mangano C, Mangano F, Piattelli A, lezzi G, Mangano A, La Colla L. Prospective clinical evaluation of 307 single-tooth morse taperconnection implants: A multicenter study. Int J Oral Maxillofac Implants, 2010; 25(2): 394-400.

34. Rousseau P. Flapless and traditional dental implant surgery: An open, retrospective comparative study. J Oral Maxillofac Surg, 2010; 68(9): 2299-2306.

35. Thomé G. Análise comparativa da resistência à deformação de implantes de hexágono externo com ou sem desenhos internos de inserção. [Tese] Campinas: Centro de Pós-Graduação/CPO São Leopoldo Mandic; 2007.

36. Pierrisnard L, Renouard F, Renouard P, Barquins M. Influence of implant length and bicortical anchorage on implant stress distribution. Clin Implant Dent and Relat Res, 2003; 5(4): 254-262.

37. Araújo CRP, Araújo MAR, Conti PCR, Assis NMSP, Maior BSS. Estudo clínico e radiográfico randomizado (RCT) prospectivo com implantes Cone-Morse. Implant News, 2008; 5(2): 191-195.

38. Oh TJ, Yoon J, Misch CE, Wang HL. The causes of early implant bone loss: Myth or science? J Periodontol, 2002; 73(3): 322-333.

39. Nisapakultorn K, Suphanantachat S, Silkosessak O, Rattanamongkolgul S. Factors affecting soft tissue level around anterior maxillary single-tooth implants. Clin Oral Implants Res, 2010; 21(6): 662 670.

40. Sorni-Bröker M, Peñarrocha-Diago M, Peñarrocha-Diago M. Factors that influence the position of the peri-implant soft tissues: A review. Med Oral Patol Oral Cir Bucal, 2009; 14(9): e475-479.

41. Chow YC, Eber RM, Tsao YP, Shotwell JL, Wang HL. Factors associated with the appearance of gingival papillae. J Clin Periodontol, 2010; 37(8): 719-727

42. Chow YC, Wang HL. Factors and techniques influencing peri-implant papillae. Implant Dent, 2010; 19(3): 208-219.

43. Maynard JG Jr, Wilson RD. Physiologic dimensions of the periodontium significant to the restorative dentist. J Periodontol, 1979; 50(4): 170-174. 44. Degidi M, Piattelli A, Gehrke P, Carinci F. Clinical outcome of 802 immediately loaded 2-stage submerged implants with a new grit-blasted and acid-etched surface: 12-month follow-up. Int $J$ Oral Maxillofac Implants, 2006; 21(5): 763-768.

45. Degidi M, Novaes AB Jr, Nardi D, Piattelli A. Outcome analysis of immediately placed, immediately restored implants in the esthetic area: The clinical relevance of different interimplant distances. J Periodontol, 2008; 79(6): 1056-1061. 